\title{
Appearance Measurement System Using Fuzzy Logic
}

\author{
Haizhuang Kang, Clive Butler, Qingping Yang, Franco Sacerdotti, Fabrizio Benati \\ Department of Systems Engineering, Brunel University \\ Uxbridge, Middlesex, UB8 3PH, UK \\ Tel: +44 1895274000 Ext. 2954, Fax: +44 1895812556 \\ Email: empghzk@brunel.ac.uk
}

\begin{abstract}
To objectively measure the paint appearance on car body as same as perceived by customers, this paper presents a novel method to model the paint appearance using fuzzy logic. Three popular parameters, Orange Peel, Metal Texture Effect and Gloss, are used as system input, overall appearance is defined as system output. This fuzzy logic model is fully based on human understanding and expertise. The preliminary result shows that this model can well mimic human behavior to reflect the correlation between overall appearance and individual properties on paint surface.
\end{abstract}

\section{Introduction}

In modern automotive industry, attractive paint appearance on car bodies is highly demanded to meet customers' satisfaction. To improve paint quality, one of the important tasks is to measure the quality of paint appearance, as perceived by customers, in a repeatable, reproducible, and continuous scale manner. A survey in this area showed that some parameters, such as the gloss, distinctness of image (DOI), and orange peel (long wave) and metal texture (short wave) have been used to evaluate paint appearance. These parameters reflect a variety of properties of the paint appearance. To correspond to human visual perception, an overall appearance index is required to be presented with combining all the information obtained from the paint surface. As this is a multi-input, non-linear problem, traditional mathematical model would be complicated to solve this sort of problem. Here a fuzzy logic model is employed to integrate the relevant parameters as an overall appearance index.

In this paper, we use Mamdani-type fuzzy logic to model the appearance measurement system. With fuzzyrule-based fuzzy logic system, the overall appearance index can be easily calculated by synthesizing a set of linguistic rules obtained from human understanding on paint appearance. The preliminary result shows that this system could effectively evaluate overall appearance with a high visual correlation.

\section{Appearance Measurement}

\subsection{The definition of appearance}

What is the appearance? ASTM (American Society for Testing and Materials) E284 gives two general definitions:

1 The aspect of visual experience by which things are recognized. (1990)

2 In psychophysical studies, visual perception in which the spectral and geometric aspects of a visual stimulus are integrated with its illuminating and viewing environment. (1993)

Dr. Deane B. Judd at the National Bureau of Standard (NBS, USA) in 1934 proposed a classification of the optical attributes which combine to identify the appearances of objects and materials. Three principal modes of appearance related to the geometric conditions of illumination and view are ${ }^{[1]}$ :

\section{Mode-1 Aperture}

The color is seen through a hole so that the observer is unable to identify the illuminant or objects involved or both. He can measure only the distribution of energy passing through the aperture.

\section{Mode-2 Illuminant}

For lights and other self-luminous objects, spectral and geometric distributions of light can be measured.

Mode-3 Object

The observer is conscious of both the object seen and its environment. 
Our primary concern is with Mode-3, the object mode. Based on the Mode 3, Richard S. Hunter presented four submodes of the object mode ${ }^{[2]}$.

1. Opaque, reflecting nonmetallic objects: paint films, molded plastic objects, thick textile fabrics, papers, and so forth.

2. Opaque metallic objects (bare metal surfaces): automobile bumpers and metallic trim.

3. Translucent (light-transmitting and reflecting) objects.

4. Transparent (clear) light-transmitting objects.

Hunter placed the concern on the submode-1, "Opaque, Reflecting Non-metallic Objects". Although the object is primarily metal, optically it is the opaque coating over the metal that establishes the appearance and, therefore, its geometric submode, which is opaque nonmetal. $\mathrm{He}$ thought that the dominant appearance attributes are the color (chromaticity and lightness) by diffuse reflection and the gloss by specular reflection. The model, submodel, and submodel-1's attributes are depicted in Figure 1.

In automotive industry, as the paint quality can achieve a high level now, the appearance measurement is mainly emphasized on the parameters of specular gloss, orange peel and metal texture effect. As specular gloss is a standard parameter, which can be found details in reference [3], here we only discuss very common phenomena in automotive industry, orange peel and metal texture.

\subsection{Oringe Peel and Metal Texture}

There is no standard definition for both orange peel and metal texture effect. In automotive industry, Orange peel is often described as a reflected wavy light/dark pattern on the paint surface that can be seen with our naked eyes due to the interference of multiple layers of coatings (Figure 2) and steel texture.

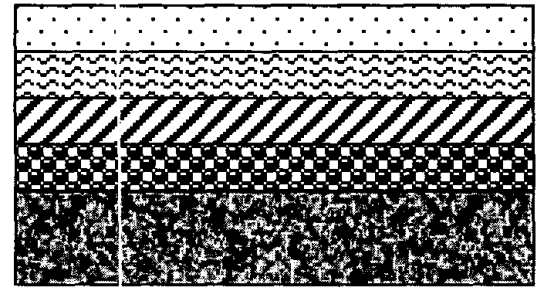

Clear Coat

Base Coat

Primer

E-Coat

Steel Sheet

Figure 2. Paint Surface structure

In this paper, this reflected wavy pattern is interpreted into two terms 'orange peel' and 'metal texture'. Orange peel is referred to large pattern (long wave, wavelength between $1-10 \mathrm{~mm}$ ) due to paint/coating properties (viscosity, paint flow, thickness of film, and whether the coating is waterborne or solvent based) and painting procedure (the distance of the paint gun/bell from the product, fluid flow, temperature and humidity conditions,

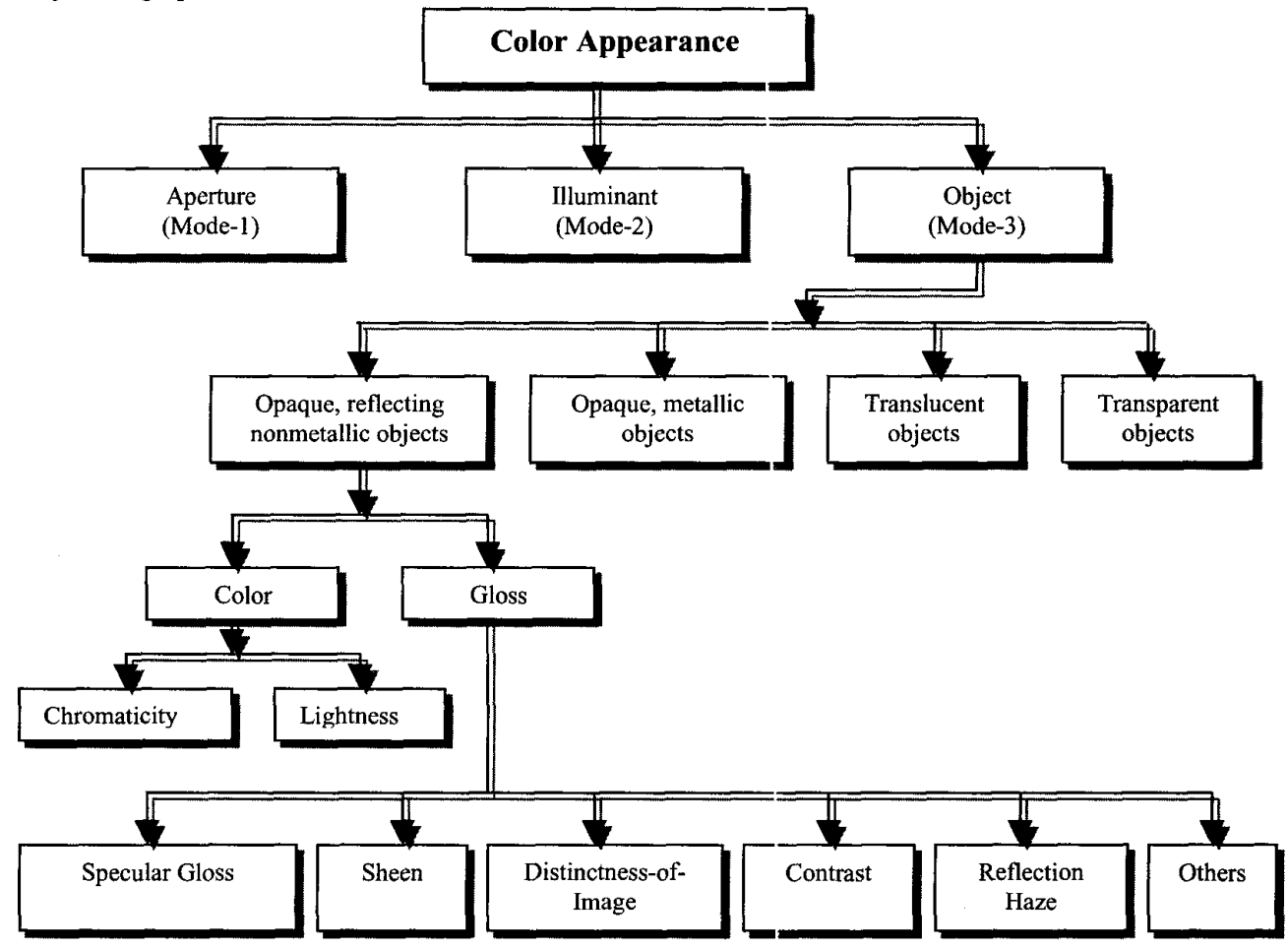

Figure 1. Models, Sub-models and Attributes of Color Appearance 
baking position and conditions). Metal texture effect is small pattern (short wave, wavelength between $0.1-1 \mathrm{~mm}$ ) which is completely caused by surface topography roughness and waviness.

The orange peel and metal texture can be easily observed with the aid of fluorescent tube reflected on the painted panel. Figure 3 (a) is the case of orange peel dominated panel. The metal texture is obscure. (b) is the case of metal texture only panel. There is no orange peel, but metal texture is obvious around the tube.

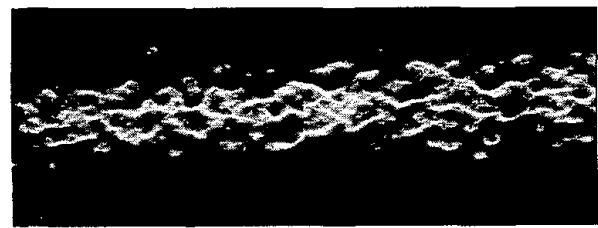

(a)

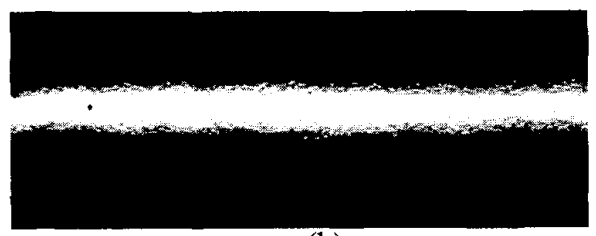

(b)

Figure 3. Orange peel and Metal texture

What is the common and difference between orange peel and metal texture effect?

\section{Common:}

a. Both of them are not physical profiles of the surface, but sort of optical reflected phenomenon on the painted surface that can be seen with our naked eyes with the aid of light source.

b. They all have effects on the paint appearance.

Difference:

a. The cause is different. Orange peel is often thought derived from paint/coating properties and painting procedure. Metal texture effect is completely caused by surface topography.

b. The effect is different. The wavelength of orange peel is larger than that of texture effect. The paint appearance is more likely sensitive to orange peel effect.

The relation between orange peel and metal texture is that the steel texture can affect the paint procedure to cause orange peel. But it is not critical factor for orange peel. Large orange peel could occur due to improper control of paint procedure even if steel texture is very smooth. Large orange peel can obscure metal texture effect. In this case only orange peel is evaluated.

\subsection{Parameter measurement}

The parameter measurement in this case mainly involved in measurement of specular gloss, orange peel, and metal texture. The measurement of specular gloss has been well-established ${ }^{[3][4]}$ and commercial product can be obtained from market. The orange peel, and metal texture measurement are far away from standard. There are several methods developed for this purpose. The most used commercial instrument is Wavescan (BYK, Gardener), which measure long wave and short wave ${ }^{[5]}$. Author also developed a method to measure orange peel and metal texture using image signal processing ${ }^{[6]}$, which can effectively evaluate the effect from orange peel and metal texture on painted panels. All the details can be referred to these references.

\subsection{Appearance Index}

Appearance index is quantified overall assessment of paint quality. It is related with all individual parameters. Some empirical formula gives each parameter an index to present the overall appearance like:

\section{$A I=0.15$ Gloss +0.35 DOI +0.25 Orange Peel +0.25 Contrast Loss}

Actually the relation between appearance index and individual parameters is more complicated. Because this is a multiple input and non-linear system. It also involved human's psychoanalysis. It is difficult to model this system with traditional mathematics method.

For human visual assessment, we can give a quick assessment by comparing the parameters like gloss, orange peel and metal texture, and recalling the previous knowledge. Here we try to impose human understanding, especially expertise to this measurement system. For example, based on previous study, we found that if gloss value is higher than 80 , there is no direct correlation between gloss and AI; if gloss is lower than 60, the methods for measuring orange peel and metal texture would be invalid; if gloss between 60 to 80 , the AI can be directly affected by gloss. Another relation between orange peel and metal texture is that if orange peel is higher than 40 , metal texture can be ignored, because orange peel has already dominated appearance. How to add these understandings into the system and set up a reliable measurement system acting like our human brain, it is what we try to resolve in this paper. 


\section{Fuzzy-Logic modeled System}

\subsection{Why use Fuzzy Logic?}

Fuzzy logic is all about the relative importance of precision. It is a convenient way to map an input space to an output space, which is cheaper and faster. In this case, particular observations about fuzzy logic are:

- Fuzzy logic can built on the experience of experts. As overall appearance index is required to relate human visual perception, human understanding on assessment of paint appearance should be the basis of the measurement system. Built on experts' experience, the system can be more reliable and objective

- Fuzzy logic is an open, flexible system which let you change the system performance with newly knowledge obtained.

- Fuzzy logic can model non-linear functions of arbitrary complexity. As this system is multi-input and non-linear, fuzzy logic can easily map the input and output.

- Fuzzy logic is conceptually easy to understand and use over traditional mathematical method, because it is based on natural language of human communication which underpins many of the other advantages of fuzzy logic.

\subsection{Mamdani-type Fuzzy Model}

Mamdani's fuzzy inference method is the most commonly seen fuzzy methodology. Ebrahim Mamdani proposed it in 1975 as an attempt to control a steam engine and boiler combination by synthesizing a set of linguistic control rules obtained from experienced human operators [7]. In this case, a Mamdani-type fuzzy logic is chosen to model the appearance measurement system ${ }^{[8]}$ (Figure 4).

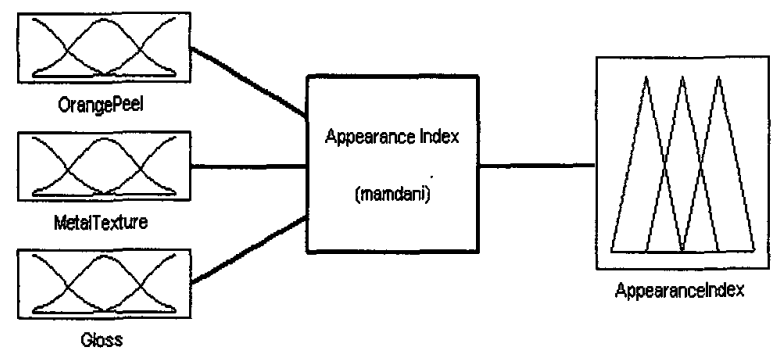

Figure 4. Mamdani model for Appearance Index
There are three inputs, orange peel (OP), metal texture (MT) and gloss, and one output, Appearance Index (AI). The fuzzy' logic system is based on eight rules.

Input- 1 is orange peel which limited between 0 to 100 (Figure 5(a)). There are three Gauss member functions representing low, middle and high value according to human visual perception. Low value means orange peel effect is small, appearance looks good. When the value of orange peel higher than 50 , the appearance could be badly damaged. So the middle function is set on 30 . The similar situation is in the input-2, metal texture (Figure 5(b)) which the only difference is the parameters of member function. As the metal texture effect is less than orange peel, the middle function is set on 40 . Input- 3 has only two member functions corresponding to low and high value (Figure $5(\mathrm{c}))$. This is because if gloss is higher than 80 , the appearance relies on other inputs. If gloss were between 60 and 80 , appearance would drop quickly. If gloss is lower than 60 , the orange peel and metal texture can not be measured with this method. So appearance is thought to be very poor. The output has four member functions representing four conditions of appearance, perfect, good, average, and poor (Figure $5(\mathrm{~d})$ ).
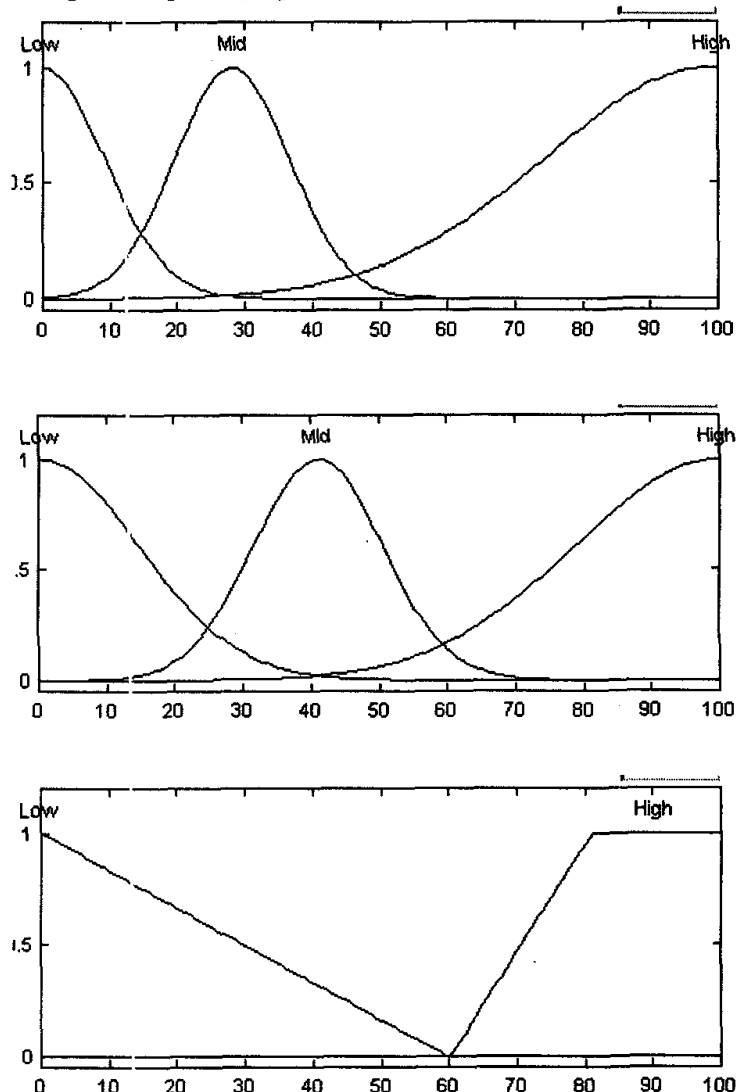


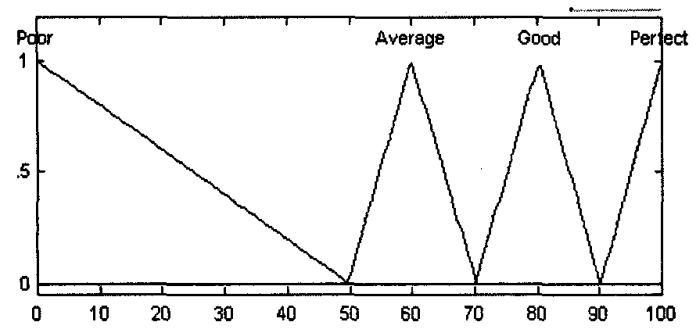

Figure 5. Member functions in input and output

The process of how this fuzzy logic system works is shown in Figure 6. The defuzzification method is the centroid calculation, which returns the center of area under the curve of output.

Based on previous knowledge and expertise, eight rules are used to evaluate in parallel for fuzzy reasoning. They are:

1. If [orange peel is low] and [metal texture is low] and [gloss is high] then [Appearance Index is perfect]
2. If [orange peel is low] and [metal texture is Mid] and [gloss is high] then [Appearance Index is good]

3. If [orange peel is low] and [metal texture is high] and [gloss is high] then [Appearance Index is average]

4. If [orange peel is mid] and [metal texture is low] and [gloss is high] then [Appearance Index is good)

5. If [orange peel is mid] and [metal texture is mid] and [gloss is high] then [Appearance Index is average)

6. If [orange peel is mid] and [metal texture is high] and [gloss is high] then [Appearance Index is poor)

7. If [orange peel is high] then [Appearance Index is poor]

8. If [gloss is low] then [Appearance Index is poor]

With these eight rules, an example is given to illustrate the reasoning procedure of fuzzy logic. In Figure 7 , the orange peel is 10.8 , metal texture equals 27.7 and gloss is 86.7. The output, appearance index is 76.4 .

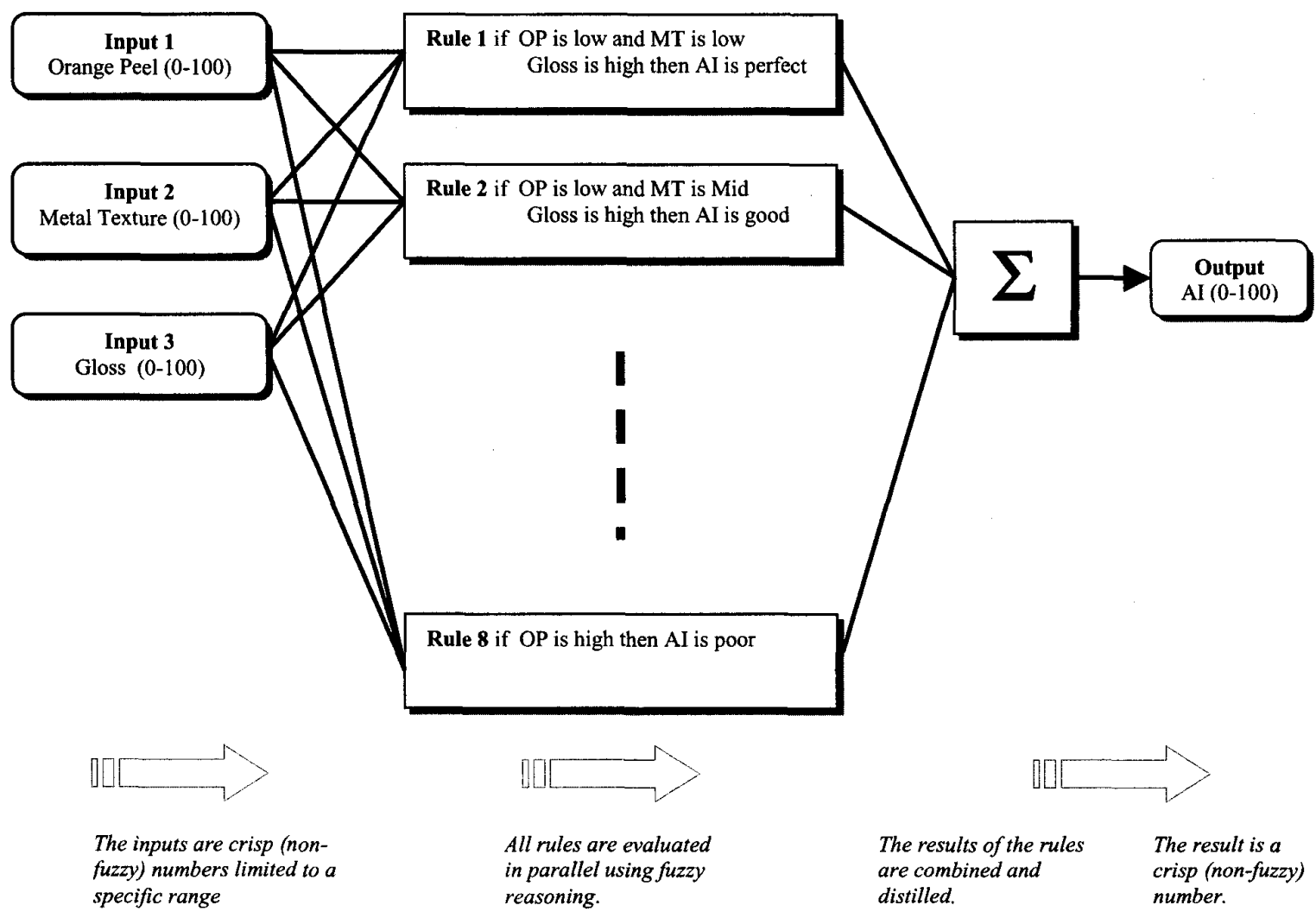

Figure 6. Working process of fuzzy 


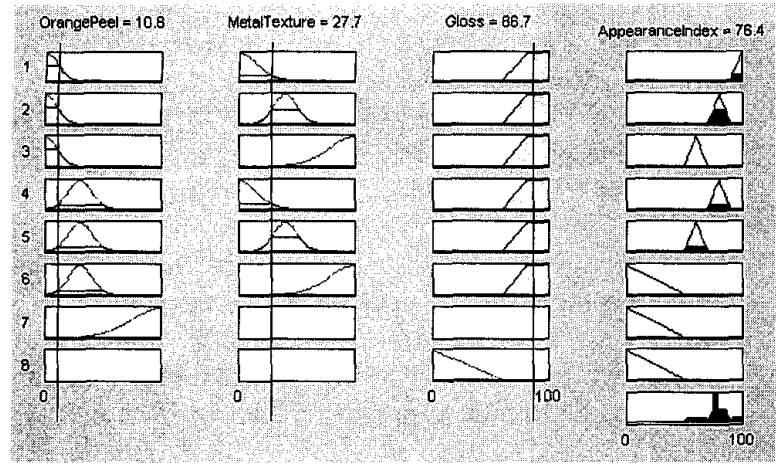

Figure 7. An example of fuzzy logic reasoning procedure

In automotive industry, the gloss of paint surface is normally higher than 80 . If setting gloss at 80 , a 2 -D surface can be drawn to display the relation of appearance index with orange peel and metal texture (Figure 8).

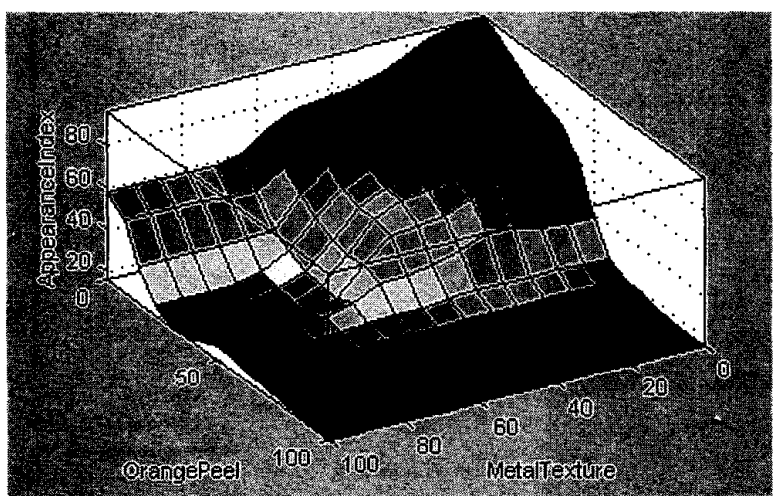

Figure 8. Appearance index surface

\section{Result and Conclusion}

This model has been testified with many panels. Here a test result is given with ten panels, which were specially selected with a range of orange peel and metal texture. Figure 9 shows the relation between AI and visual assessment. The method for visual assessment can be referred to reference ${ }^{[9]}$, the measurement of orange peel and metal texture can referred to reference ${ }^{[6]}$.

With this model, the measured gloss, orange peel and metal texture on these panels can be combined to produce an appearance index. Form the above result, it can be seen that $\mathrm{AI}$ has a strong correlation with visual perception $\left(\mathrm{R}^{2}=0.925\right)$. So it can well represent visual perception.

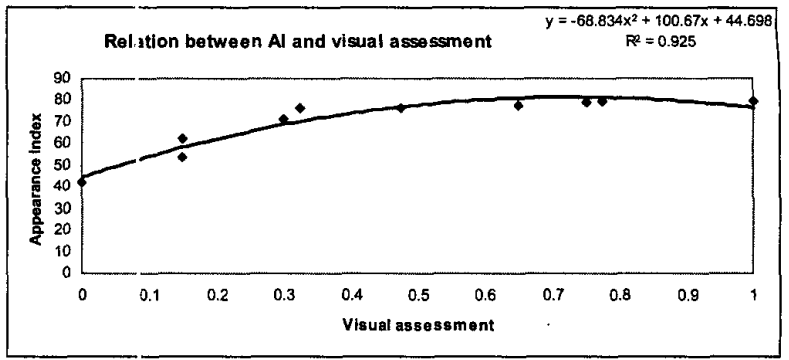

Figure 9. Relation between $\mathrm{Al}$ and visual assessment

From the description above, the conclusion can be drawn that fuzzy logic system is a cheap and easy-to-use system. But it is powerful to solve any non-linear problem. Appearance index can be effectively calculated with this system. It is also an open system, it can be consummated with increasing knowledge on paint quality understanding. It is expected that an integrated system could be developed to measure all the relevant parameters and produce an overall appearance index.

\section{Reference:}

[1] B. J. Judd, A Five-attribute System of Describing Visual Appearance, STP 297, American Society for Testing and Materials, Philadelphia, 1961.

[2] R. S. Hunter, The Modes of Appearance and Their Attributes, Review and Evaluation of Appearance: Nethods and Techniques, ASTM STP 914, J. J. Rennilson and W. N. Hale, Eds. , American Society for Testing and Materials, Philadelphia, 1986, pp.5-13.

[3] K. B. Smith, A Sharp Look at Gloss, Surface Coatings International 1997 (12).

[4] ISO 2813: 1994(E), Third edition, Paints and vornishes-Determination of specular gloss of nonmetallic paint films at $20^{\circ}, 60^{\circ}$ and $85^{\circ}$.

[5] Gabriele Kigle-Boeckler, Surface smoothness and its influence on paint appearance-How to measure and control it, ANTEC '96, pp3433

[6] H. Kang, C. Butler and Q. Yang, Measurement of Paint Appearance Using Image Processing System, IEEE International Workshop on Intelligent Signal Processing, Budapest, Hungary, Sep. 1999

[7] E.H. Mamdani and S. Assilian, An experiment in linguistic synthesis with a fuzzy logic controller, International Journal of Man-Machine Studies, Vol. 7, No. 1, pp. 1-13, 1975

[8] Fuzzy Logic Toolbox User's Guide, The MathWorks, Inc. 1999

[9] H. Kang, C. Butler, Q. Yang, F. Sacerdotti, F. Benati, Visual Assessment of Paint Appearance on Car Body Surface, X. International Colloquium on Surfaces, Chemnitz, Germany, 2000 